\title{
Full-mouth Rehabilitation of a Patient with Severe Attrition using Hobo Twin-Stage Procedure
}

\author{
${ }^{1}$ Saurav Banerjee, ${ }^{1}$ Nabarun Chakraborty, ${ }^{1}$ Rajwinder Singh, ${ }^{2}$ Tapas Gupta, ${ }^{3}$ Ardhendu Banerjee \\ ${ }^{1}$ Postgraduate Student, Department of Prosthetic Dentistry, Dr R Ahmed Dental College and Hospital, Kolkata, West Bengal, India \\ ${ }^{2}$ Professor, Department of Prosthetic Dentistry, Dr R Ahmed Dental College and Hospital, Kolkata, West Bengal, India \\ ${ }^{3}$ Professor and Head, Department of Prosthetic Dentistry, Dr R Ahmed Dental College and Hospital, Kolkata, West Bengal, India
}

Correspondence: Saurav Banerjee, Postgraduate Student, Department of Prosthetic Dentistry, Dr R Ahmed Dental College and Hospital, Kolkata, West Bengal, India, e-mail: sauravbnrj@gmail.com

\section{ABSTRACT}

Excessive occlusal wear can result in pulpal injury, occlusal disharmony, impaired function and esthetic deformity. Loss of anterior guidance can result from severe wear of anterior teeth, which protects the posterior teeth during excursive movement. The collapse of posterior teeth also results in the loss of normal occlusal plane and the reduction of the vertical dimension. This clinical report describes the use of Hobo Twinstage procedure for rehabilitation of a patient with severe tooth wear, resulting in reduced VDO.

Keywords: Anterior guidance, Hobo twin stage, Lucia jig, Wear.

\section{INTRODUCTION}

Gradual wear of the occlusal surfaces of teeth is a customary process during the lifetime of a patient. However, excessive occlusal wear can result in pulpal injury, occlusal disharmony, impaired function and esthetic deformity. ${ }^{1}$

Tooth wear can be classified as attrition, abrasion, and erosion and leads to an alteration of the vertical dimension of occlusion (VDO). In many cases, the VDO is maintained by tooth eruption and alveolar bone growth. As teeth are worn out, the alveolar bone undergoes an adaptive process and compensates for the loss of tooth structure to maintain the V D 0 . Therefore, VDO should be changed carefully. Increasing the VDO in bruxers puts a severe overload on the teeth and often results in the destruction of the restorations or teeth themselves. ${ }^{2,3}$

This clinical report highlights the use of Hobo twin-stage procedure for rehabilitation of a patient with severe tooth wear, resulting in reduced VDO. In this procedure, a cast with a removable anterior segment was fabricated and occlusal morphology of posterior teeth was reproduced without anterior segment. This was done to produce a cusp angle coincident with the standard values of effective cusp angle (condition 1). Then reproduction of anterior morphology was done with the anterior segment and providing anterior guidance which produces a standard amount of disocclusion (condition 2 ) $^{4}$ (Table 1).

\section{CASE REPORT}

A 54-year-old male reported to the department of prosthodontics with a chief complaint of difficulty in chewing food and sensitivity to hot and cold food items (Figs 1 and 2). The patient gave no significant medical history and did not report any signs of temporomandibular joint disorder or myofascial pain dysfunction.

Extraoral examination depicted no facial asymmetry, or muscle tenderness. The mandibular range of motion was within normal limits. Intraoral examination establ ished grossly attrited dentition in both maxillary and mandibular arches. Both the arches were fully dentate with spacing between maxillary and mandibular anterior tooth. The patient had a bilateral class I molar relation and a canine-guided occlusion. A pproximately $3 \mathrm{~mm}$ of loss in vertical dimension of occlusion was established. Full mouth reconstruction with Hobo twin-stage technique was planned to reconstruct the attrited dentition in functional harmony to the stomatognathic system while providing a canine-guided disocclusion during eccentric movements. A n

Table 1: Articulator adjustment values for Hobo twin-stage procedure (degree)

\begin{tabular}{lccccc}
\hline Condition & \multicolumn{2}{c}{ Condylar path } & & \multicolumn{2}{c}{ Anterior guide table } \\
\cline { 2 - 5 } & $\begin{array}{c}\text { Sagittal condylar } \\
\text { path inclination }\end{array}$ & Bennett angle & & Sagittal inclination & Lateral wing angle \\
\hline Condition 1 without anterior teeth & 25 & 15 & 25 & 10 \\
Condition 2 with anterior teeth & 40 & 15 & 45 & 20 \\
\hline
\end{tabular}




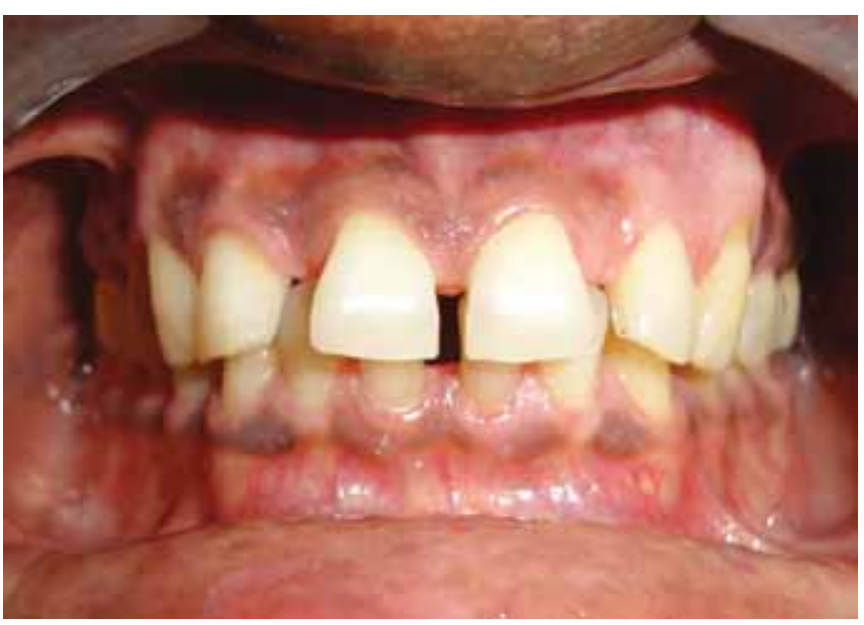

Fig. 1: Preoperative intraoral view in occlusion
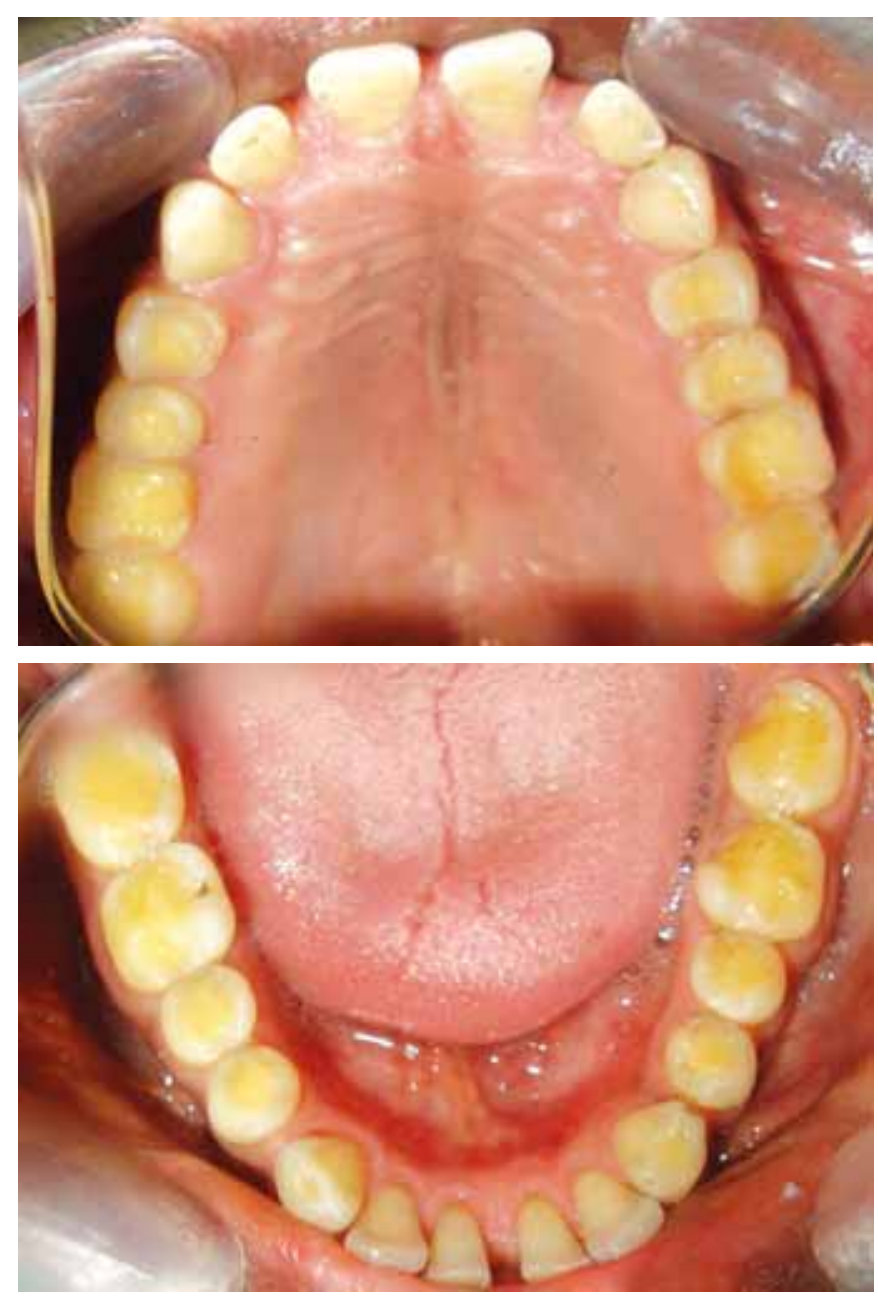

Fig. 2: Preoperative intraoral occlusal view

increase of $3 \mathrm{~mm}$ of vertical dimension was also planned. The amount of bite rise to be achieved was al so evaluated using the 'Closest S- speaking space' or the 'F reeway space' technique.

\section{PROCEDURE}

1. Consent was taken from the patient before the start of treatment. Impressions of both arches were made with irreversible hydrocolloid material (Tropicalgin, Zhermack, Italy) and diagnostic casts were obtained.

2. The maxillary cast was mounted on a semi-adjustable articulator (W hip Mix) with a facebow transfer and mandibular cast was mounted with a Lucia Jig in the anterior region and interocclusal records (B itrex, Equinox, $\mathrm{N}$ etherlands) in the posterior region.

3. An occlusal splint (Heat-cured acrylic resin, DPI, M umbai.) was provided to the patient as part of reversible interventional modalities to evaluate adaptation of the patient to altered VDO. The patient was kept in diagnostic and observational period of 6 weeks before the definitive restorative phase of rehabilitation was started.

4. A diagnostic wax-up of the full mouth restoration was carried out at the increased vertical dimension for posterior teeth without the anterior segment of maxillary cast in place. To produce standard effective cusp angles, the condylar and the incisal guidance were set to condition 1 . At this position the diagnostic wax-up was balanced in protrusive excursion and lateral excursions. The anterior segment of the cast was reassembled and the condylar guidance and incisal guidance were set again (condition 2) and the wax-up was completed so as to generate posterior disocclusion (Fig. 3).

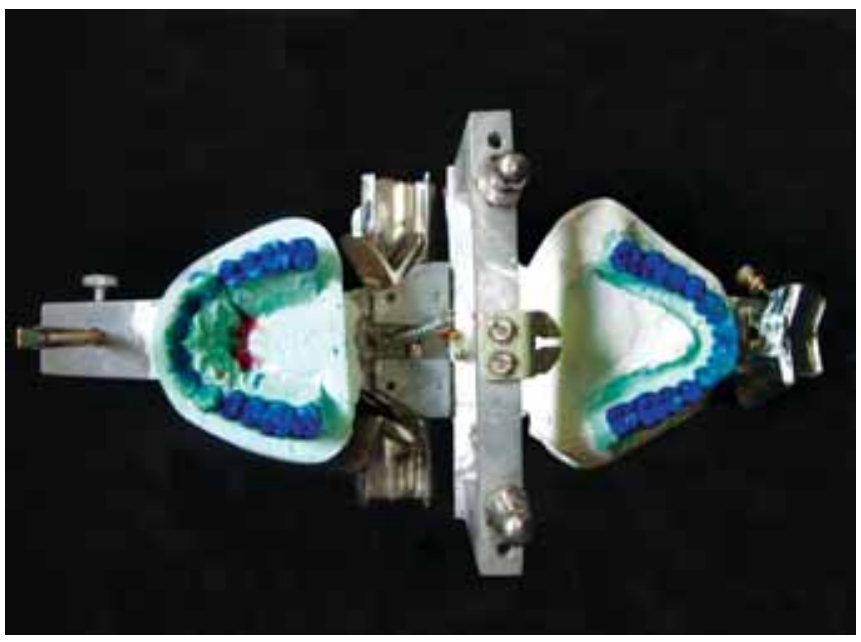

Fig. 3: Diagnostic wax-up

5. Teeth number $16,17,26,27,36,37,46$ and 47 were prepared for full coverage metal restoration while remaining teeth were prepared for metal-ceramic restorations. Stage I temporary restorations (DPI dental products, M umbai) were fabricated chairside quadrant by quadrant during several appointments to minimize patient discomfort. The patient's vertical dimension of occlusion was maintained by using unprepared second molar teeth as occlusal vertical stops, which will be prepared later. Minimal occlusal reduction is indicated for patients scheduled for rehabilitation at an altered vertical dimension of occlusion. 
6. Then the second molars were prepared and stage II temporaries (DPI dental products, Mumbai) were fabricated using the index of the diagnostic wax-up and cemented with ZnO noneugenol cement (Freegenol, GC, India) and left for 3 weeks.

7. Once the patient was adapted to this position, a final full arch impression for maxillary and mandibular teeth was made using poly (vinyl siloxane) impression material (Reprosil, Dentsply, USA), and casts were poured in type IV gypsum (Kalabhai Karson, M umbai). This cast was mounted on Whip-M ix articulator using the facebow transfer.

8. Now to transfer the vertical dimension and centric relation, temporaries were removed from both maxillary and mandibular left posterior region while the temporaries of right and anterior maxillary and mandibular region acted as a stop. Interocclusal recording material was injected between the left maxillary and mandibular prepared tooth. Likewise, the temporaries were removed from right maxillary and mandibular region while the temporaries were present in left, an anterior region of both arches, interocclusal record was injected between the right maxillary and mandibular prepared tooth, the same procedure was followed in anterior region. The three segmental interocclusal records thus obtained were used to mount the mandibular cast.

9. The wax pattern was fabricated with the anterior mandibular segment removable following condition 1 and 2 (Figs 4 to 6). All the wax patterns were cast, and metal copings (K eraN, Germany) were tried in patient's mouth. Definite restorations with PFM crowns exhibiting a vital and natural appearance with proper contour and shade were fabricated.

10. Permanent cementation was done with GIC luting cement (K etac Cem, 3M ESPE, Germany). Oral hygiene instructions emphasizing use of dental floss and proper brushing were given and follow-up was carried out at a interval of 6 weeks (Figs 7 to 11).

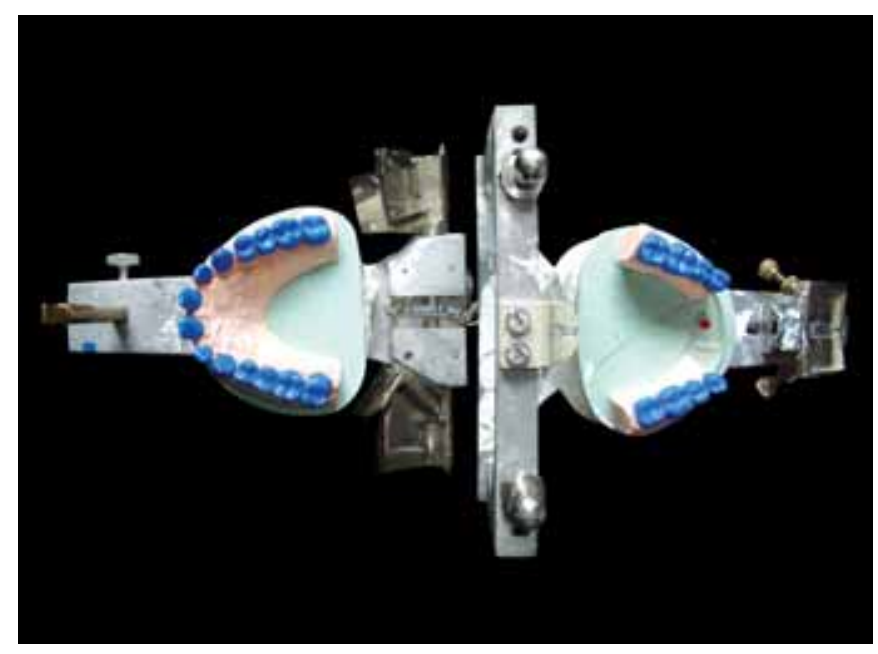

Fig. 4: Wax-up after condition 1

\section{DISCUSSION}

Function and health can be restored for worn out dentition using Hobo twin-stage procedure. There has been conflicting opinion whether to work simultaneously or to work on different segments of the arch individually. The proponents of the later theory states that work can be completed more quickly and easily and with much more comfort for the patient. The total

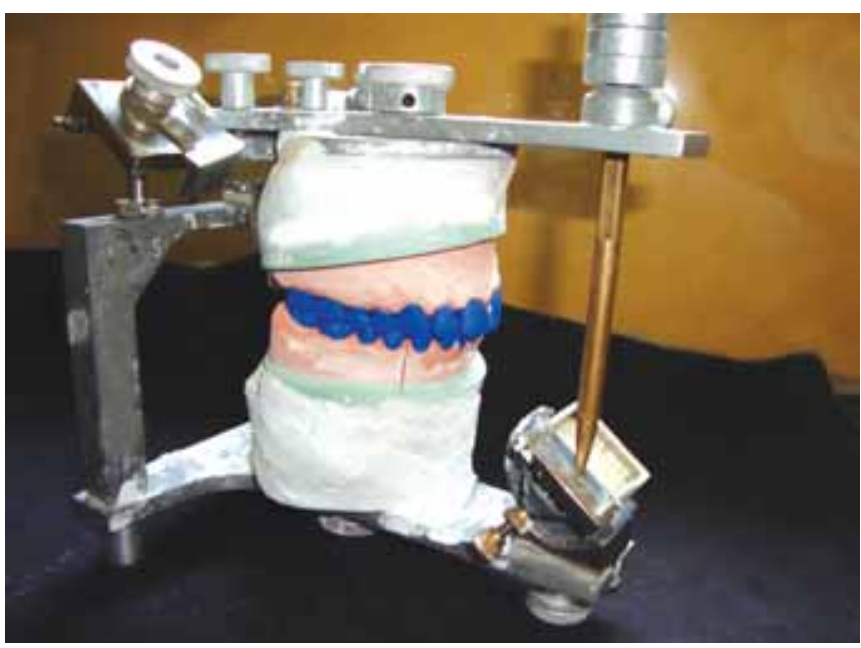

Fig. 5: Wax pattern after condition 2

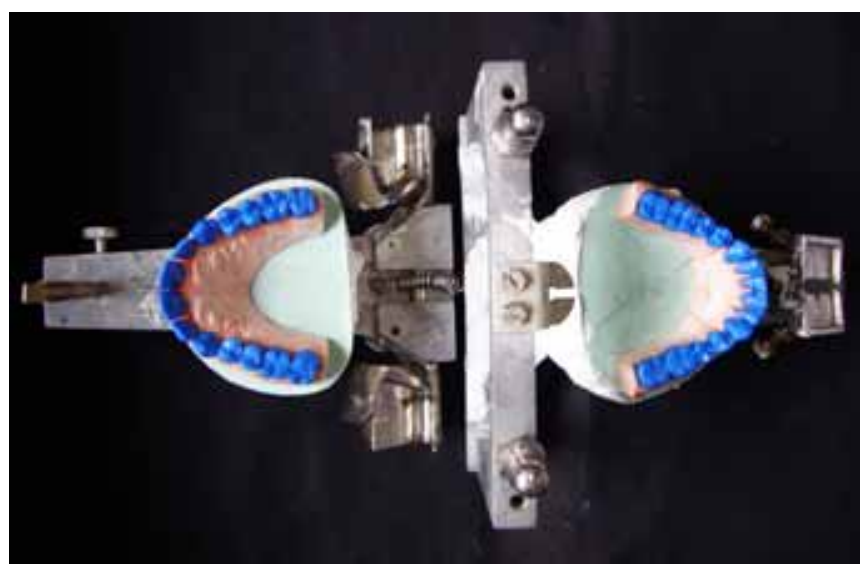

Fig. 6: Wax pattern after condition 2

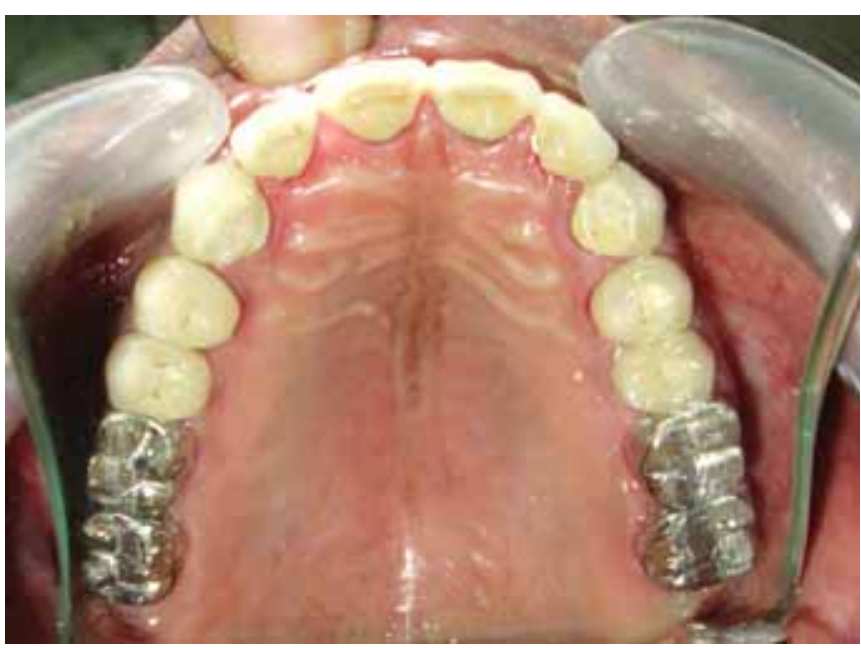

Fig. 7: Postoperative intraoral view of maxillary arch 


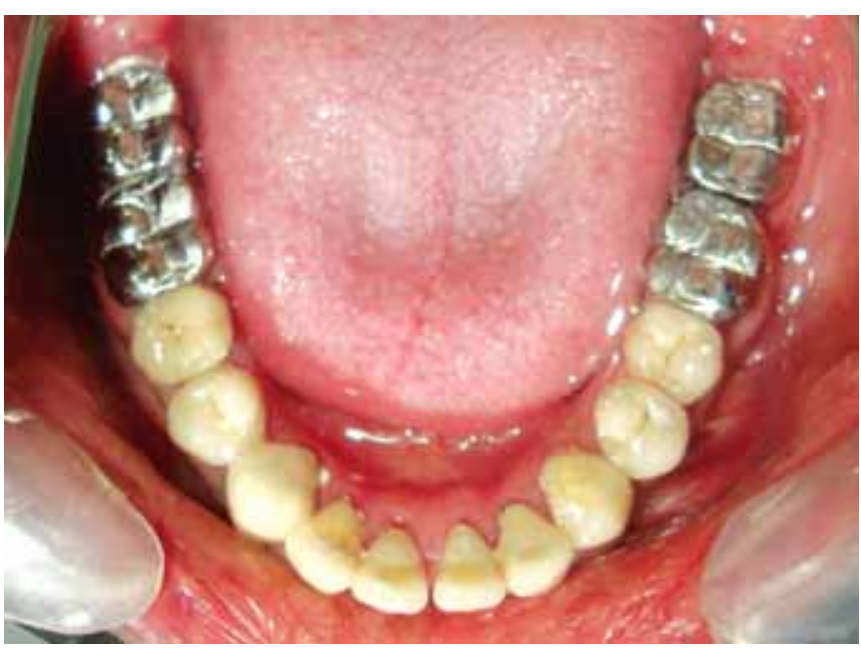

Fig. 8: Postoperative intraoral view of mandibular arch

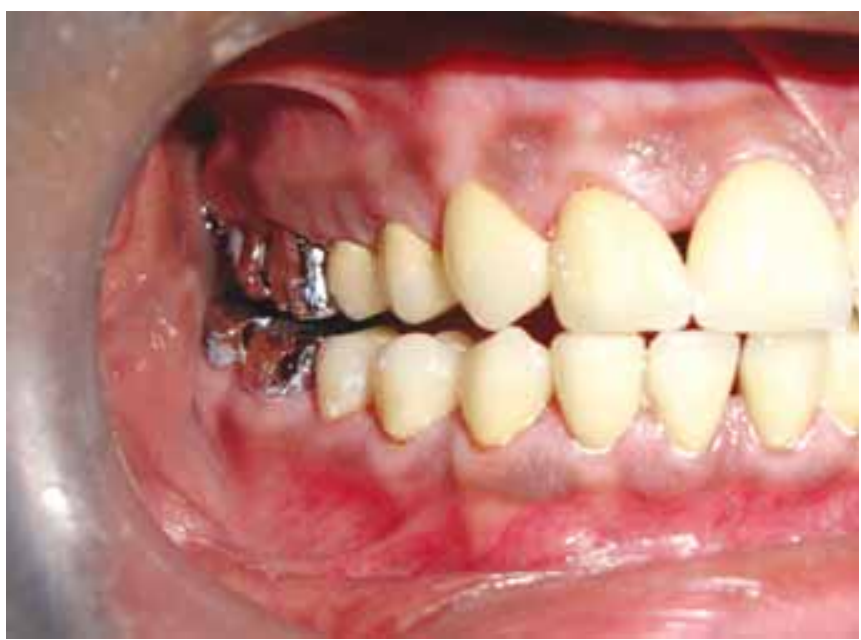

Fig. 9: Canine-guided disocclusion during right lateral movement

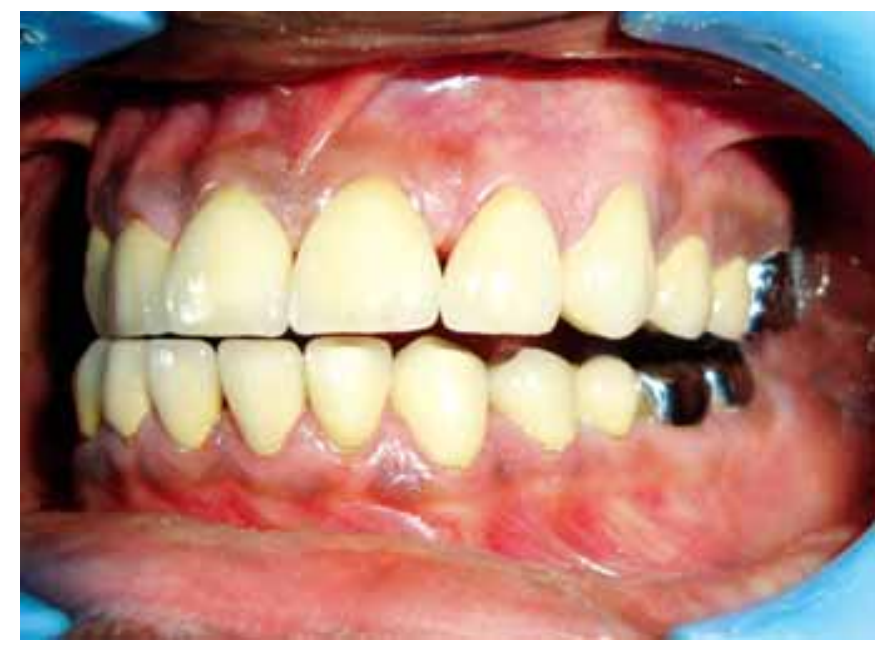

Fig. 10: Balancing side disocclusion during right lateral movement

chairside and laboratory time for rehabilitation is significantly reduced. ${ }^{5}$ The disadvantages include restrictions for achieving ideal occlusion when altering the vertical dimension, occlusal plane.

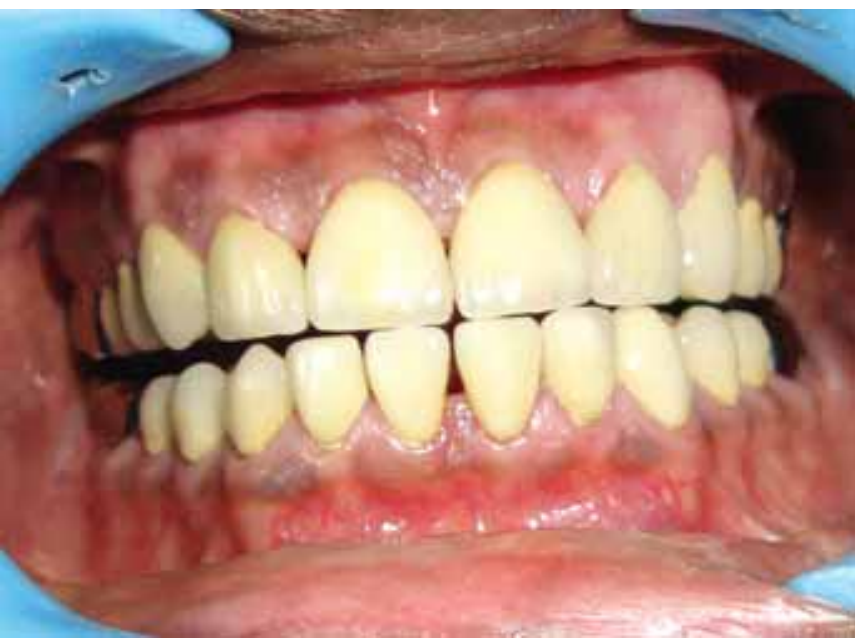

Fig. 11: Protrusive disocclusion

Previously, the condylar path was the principle focus of attention for gnathologists, since it did not change during adul thood and the determination of anterior guidance remained the sole discretion of the dentist. Thus, anterior guidance and the condylar path were considered independent factors. Dawson stated that the condylar path was not a determination of anterior guidance, and that it did not matter whether the anterior path was flat, curved, concave, convex or parabolic, the rotating condyle sliding down the unchanged condylar path permits the lower anterior teeth to follow any number of path variations without interference. ${ }^{6}$ While in Pankeymann-Schuyler philosophy anterior guidance is first established followed by restoration of the posterior teeth. Functional generated path technique is used to assist waxing of upper posterior restorations but movement of teeth while making FGP compromised registration. A rea of freedom between centric relation and intercuspal position was also present. ${ }^{5}$

Recent concepts revealed that anterior guidance influences the working condylar path and even changes when the lateral incisal path deviates from the optimal orbit and supports the hypothesis that anterior guidance and the condylar path are dependent factors. In setting anterior guidance, it is recommended to set the working condyle so that it moves straight outward al ong the transverse horizontal axis.

In healthy patients, anterior guidance is about 5 degrees steeper than the condylar path in the sagittal plane. ${ }^{7} \mathrm{~W}$ hen a patient protrudes the mandible, the anterior teeth guide it downward, creating space between the posterior teeth referred to as posterior disclusion. The same phenomenon occurs during lateral movement because the lingual inclination of the maxillary canine is steeper than the condylar path.

The angle of hinge rotation produced by the angular difference between anterior guidance and the condylar path assists posterior disocclusion but is not solely accountable. The angle of hinge rotation contributed to posterior disocclusion for protrusive movement was $0.2 \mathrm{~mm}$ on average and $0.5 \mathrm{~mm}$ on average for lateral movement on both working and nonworking sides. 
A ccording to an investigation on molar disocclusion during eccentric movements, the amounts of disocclusion were $1.1 \pm$ $0.6 \mathrm{~mm}$ during protrusive movement and $0.5 \pm 0.3 \mathrm{~mm}$ on the working side and $1.0 \pm 0.6 \mathrm{~mm}$ on the nonworking side during lateral movement measured at the mesiobuccal cusp tip of the mandibular first molar. ${ }^{8}$ The actual disocclusion on the working side $(0.5 \mathrm{~mm})$ was equal to the amount created by the angle of hinge rotation $(0.5 \mathrm{~mm})$. However, the actual disocclusion during protrusive and lateral movements on the nonworking side differ from the angle of hinge rotation. This leaves resi dual amounts of disocclusion unaccounted suggesting that the angle of hinge rotation was not solely responsible for disocclusion. The residual amounts can also be attributed to the cusp shape factor. $^{9}$

The anatomy of the cusps also contributes to posterior disocclusion. The posterior teeth disocclude only when the cusp inclination of the molar is parallel to the condylar path and anterior guidance is steeper than the condylar path. If the shapes of the posterior cusps are less steep than the condylar path, the posterior teeth disocclude even if anterior guidance is parallel to the condylar path.

Contraindications of the $\mathrm{H}$ obo twin-stage procedure are as follows: ${ }^{4}$

1. A bnormal curve of Spee

2. A bnormal curve of Wilson

3. A bnormally rotated tooth

4. A bnormally inclined tooth

\section{CONCLUSION}

The principles and concepts involved in oral rehabilitation using the Hobo twin-stage procedure have been discussed. The amount of disclusion of teeth is significantly controlled by the condylar and incisal guidance and disregards the role of measured condylar guidance. The average calibrations of condylar, lateral and incisal guidance and cusp angle provide an easy approach of management with lesser skills needed.

\section{REFERENCES}

1. Turner K A, M issirlian DM. Restoration of the extremely worn dentition. J Prosthet Dent 1984;52:467-74.

2. Dawson PE. Functional occlusion - from TMJ to smile design. (1st ed). N ew Y ork; Elsevier Inc 2008:430-52.

3. Jahangiri L, Jang S. Onlay partial denture technique for assessment of adequate occlusal vertical dimension: A clinical report. J Prosthet Dent 2002;87:1-4.

4. Hobo S, Takayama H. Oral rehabilitation, clinical determination of occlusion. Quintessence Publishing Co, Inc 1997:32-33.

5. M ann A W, Pankey LD. O ral rehabilitation. Part I. U se of P-M instrument in treatment planning and in restoring the lower posterior teeth. J Prosthet D ent 1960;10:151-62.

6. Dawson PE. Evaluation, diagnosis and treatment of occlusal problems. St L ouis CV M osby Co 1974:148-49.

7. $\mathrm{M} \mathrm{CH}$ orris W H. The importance of anterior teeth. J G nathology 1982;1:19-36.

8. Schuyler $\mathrm{CH}$. The function and importance of incisal guidance in oral rehabilitation. J Prosthet Dent 1963;13:1011-29.

9. Hobo S. Twin-tables technique for occlusal rehabilitation: Part IM echanism of anterior guidance.J Prosthet Dent 1991;66:299-303. 